\title{
Residents' Attitude, Knowledge, and Perceived Preparedness Toward Caring for Patients from Diverse Sociocultural Backgrounds
}

\author{
Jessie Kimbrough Marshall, Lisa A. Cooper, Alexander R. Green, Amanda Bertram, Letitia Wright, Niki Matusko, \\ Wayne McCullough, and Stephen D. Sisson ${ }^{2}$
}

\begin{abstract}
Purpose: Training residents to deliver care to increasingly diverse patients in the United States is an important strategy to help alleviate racial and ethnic disparities in health outcomes. Cross-cultural care training of residents continues to present challenges. This study sought to explore the associations among residents' cross-cultural attitudes, preparedness, and knowledge about disparities to better elucidate possible training needs.

Methods: This cross-sectional study used web-based questionnaires from 2013 to 2014. Eighty-four internal medicine residency programs with 954 residents across the United States participated. The main outcome was perceived preparedness to care for sociocultural diverse patients.

Key Results: Regression analysis showed attitude toward cross-cultural care (beta coefficient $[\beta]=0.57,95 \%$ confidence interval [Cl]: 0.49-0.64, $p<0.001)$ and report of serving a large number of racial/ethnic minorities $(\beta=0.90$, 95\% Cl: 0.56-1.24, $p<0.001$ ), and low-socioeconomic status patients ( $\beta=0.74,95 \%$ Cl: 0.37-1.10, $p<0.001)$ were positively associated with preparedness. Knowledge of disparities was poor and did not differ significantly across postgraduate year (PGY)-1, PGY-2, and PGY-3 residents (mean scores: 56\%, 58\%, and 55\%, respectively; $p=0.08$ ). Conclusion: Residents' knowledge of health and healthcare disparities is poor and does not improve during training. Residents' preparedness to provide cross-cultural care is directly associated with their attitude toward cross-cultural care and their level of exposure to patients from diverse sociocultural backgrounds. Future studies should examine the role of residents' cross-cultural care-related attitudes on their ability to care for diverse patients.
\end{abstract}

Keywords: cross-cultural care; graduate medical education; resident training; vulnerable populations

\section{Introduction}

Cross-cultural care training has been identified as an important strategy to help healthcare providers deliver higher quality care to socioculturally diverse patients. ${ }^{1}$ Sociocultural factors include race, ethnicity, primary language, income, education, religion, and other customs and values that characterize a group. ${ }^{2}$ These characteristics can play a role in shaping patient's beliefs, perceptions, and health behaviors. ${ }^{3}$ Health communication and clinical decision-making can be adversely affected, when patient and providers have differences in their sociocultural backgrounds (e.g., differences in English proficiency resulting in language barriers). ${ }^{4}$ Cross-cultural care involves the ability to provide quality healthcare and effectively communicate with diverse patients. $^{5}$ More than a decade ago, the Institute of

${ }^{1}$ Department of Medicine, University of Michigan Health System, Ann Arbor, Michigan.

${ }^{2}$ Department of Medicine, Johns Hopkins University School of Medicine, Baltimore, Maryland.

${ }^{3}$ Department of Medicine, Massachusetts General Hospital, Boston, Massachusetts.

${ }^{4}$ Office of Health Equity and Inclusion, University of Michigan Health System, Ann Arbor, Michigan.

${ }^{5}$ Division of Public Health, College of Human Medicine, Michigan State University, East Lansing, Michigan.

*Address correspondence to: Jessie Kimbrough Marshall, MD, MPH, Department of Medicine, University of Michigan Health System, 3214 Taubman Center, SPC 5376, Ann Arbor, MI 48109, E-mail: jessiekk@med.umich.edu

(c) Jessie Kimbrough Marshall et al., 2017; Published by Mary Ann Liebert, Inc. This Open Access article is distributed under the terms of the Creative Commons License (http://creativecommons.org/licenses/by/4.0), which permits unrestricted use, distribution, and reproduction in any medium, provided the original work is properly credited. 
Medicine released two landmark reports, Crossing the Quality Chasm and Unequal Treatment, which heightened the awareness of the critical role of cross-cultural care in improving quality and eliminating healthcare disparities. ${ }^{1,6}$ Yet, racial and ethnic healthcare disparities persist and will magnify as the diversity of the U.S. population continues to grow. Factors contributing to disparities at the provider level have included limited cross-cultural care skills, bias, and stereotyping. ${ }^{1}$ The changing U.S. demographics drive the need for trained physicians able to deliver high-quality care to socioculturally diverse patients. ${ }^{1,7-9}$

Residency training is an important opportunity to train resident physicians on high-quality cross-cultural care, including educational content on health and healthcare disparities. ${ }^{10}$ Previous studies of residents have shown them to not feel well prepared to provide cross-cultural care with associated factors, including limited training and role modeling. ${ }^{11,12}$ The Accreditation Council for Graduate Medical Education now mandates cross-cultural care training and evaluation for residents. ${ }^{13}$

We hypothesized that residents were poorly trained on contributors to healthcare disparities, and that their selfrated knowledge of healthcare disparities was higher than their measured knowledge. In addition, we posited that a curriculum on the definition of terms used to describe healthcare disparities could improve resident knowledge. We also believed that such a curriculum was only one step in addressing healthcare disparities, and that further studies will be needed to determine its impact on actually improving healthcare disparities. Overarching to our curriculum and this study is the Branch and Fraser conceptual framework model of cultural competency, which postulates that cross-cultural care training can potentially reduce racial and ethnic health disparities at the healthcare provider level. ${ }^{14}$

In this study, we explore the association of resident physicians' self-reported preparedness to care for diverse sociocultural patients and their attitude toward crosscultural care, in addition to knowledge of racial and ethnic health and healthcare disparities. Other explored variables included resident level of training, patient practice composition, and residency program characteristics.

\section{Methods}

Study design

We conducted a cross-sectional study of data collected from a cross-cultural care training module administered to internal medicine residents from July 1, 2013, to June 30,2014 . This e-learning module on health and healthcare disparities was written based on established concepts of curriculum development ${ }^{15}$ and disparities in health outcomes. ${ }^{1,7,10,16}$ Multiple choice (pre- and post-test) questions were developed based on the module content. Also, a self-assessment survey was developed using items from validated instruments on attitudes about cross-cultural care, preparedness to care for diverse patient populations, and behaviors in delivering care to culturally diverse patients. ${ }^{11,17}$ The selfassessment survey and pretest questions were used in the study's analysis.

\section{Study sample}

The didactic module was used by residents who subscribed to the Johns Hopkins Internal Medicine Ambulatory Curriculum (www.peaconline.org) ${ }^{18}$ during the 2013-2014 academic year. We examined respondents who self-identified as being in postgraduate years (PGYs) 1-3. The University of Michigan Institutional Review Board granted exemption to this study.

\section{Self-assessment questions: attitude,}

\section{preparedness, and behavior}

We used 18 predidactic survey questions to assess residents' attitudes, perceived preparedness, and self-reported clinical practice behaviors in delivering care to culturally diverse patients. ${ }^{11,17}$ Domains covered included clinic volumes of patients from racial/ethnic minority groups and low-socioeconomic status (SES), attitudes about practicing among diverse populations, consideration of culture when providing care, self-assessment of cultural sensitivity and unconscious racial bias, self-assessment of preparedness to take care of sociocultural diverse patients, and self-rated behaviors in delivering patient- and familycentered care (Supplementary Appendix 1).

\section{Pretest questions for knowledge assessment}

Twelve pretest questions were developed by internal medicine faculty with expertise in cross-cultural care, health and healthcare disparities, curriculum development, and web-based learning. Clinical vignettes and questions were modeled on existing resources. ${ }^{1,3,7,10,16,19-23}$ The questions covered a range of topics in cross-cultural care, including caring for patients with language barriers, limited literacy, low-SES, and those from differing racial and ethnic minority groups. Questions were categorized as definition/recall, comprehension, and/or application (Supplementary Appendix 2). One question was not studied because of poor item discrimination. 


\section{Study variables}

Residency program characteristics included the program's classification as university based, community based, or military based. Programs regional location in the United States was grouped into one of four regions: Mid-West, West, South, or East (Supplementary Appendix 3). ${ }^{24}$ Programs were classified as being in either an urban (location in a city/town with a population of 50,000) or nonurban location (population less than 50,000$).{ }^{25}$ PGYs $1-3$ level was used to categorize the residents' year of training. Resident knowledge of cross-cultural care that is focused on health and healthcare disparities was represented by the percentage of correct responses for both the overall pretest and the limited pretest composed of the seven questions that were focused on comprehension and application.

The set of questions related to attitude, preparedness, and behavior were assessed for correlation and reliability. Principal components analysis (PCA) was conducted on the five attitude, six preparedness, and five behavior items, respectively, to assess for correlation. Cronbach's alpha was used to measure the reliability of the items within each set of questions. After confirmation of correlation and reliability, three scales were created: attitude, preparedness, and behavior. The scales were created by summing up the corresponding question's response (each rated 0-100) within each set and then divided by 100. The scales were made to combine correlated information rather than having multiple variables measuring similar characteristics. For example, a high rating for the attitude scale represented an overall more positive attitude toward cross-cultural care.

\section{Main outcomes}

The main outcome was the self-reported preparedness scale that measured the residents' perceived preparedness to care for patients from sociocultural diverse backgrounds.

\section{Secondary outcomes}

A secondary outcome was knowledge of cross-cultural care with focus on health and healthcare disparities, as represented by the residents' percentage of correct responses for the overall pretest.

\section{Statistical analysis}

Descriptive statistics were used to assess the percentage of residents' program classification, regional designation, location in an urban or nonurban setting, and the resident PGY level. Responses for all self-assessment questions were placed on a rating scale of 0 (low)-100 (high). Pre- test responses were dichotomized to correct or incorrect and then tabulated for a composite score that was presented as the percentage of correct responses. A oneway ANOVA was used to compare pretest scores between the residents' three PGY levels. Linear regression analysis was performed to explore the association between preparedness with resident attitude, knowledge, clinical practice composition (of patients from racial and ethnic minority backgrounds and lower SES), PGY level, and residency program characteristics: program type, regional designation, and location in an urban or nonurban setting. A second linear regression model was used to explore associations between the residents' knowledge assessment score with their PGY level, attitude, and preparedness. Statistical analyses were performed using Stata/LP, version $13 .^{26}$

\section{Results}

Characteristics of study population

Eighty-four out of 158 internal medicine residency programs (52.8\%) participated in 29 states across the United States. Of the 1074 respondents who completed the module, 954 identified as being PGY-1, -2 , and -3 . The remaining 120 respondents were 10 PGY-4 and PGY-5 residents, 7 attending physicians, and 86 selfidentified "other" respondents.

The majority of residents came from a university-based program, 619 (64.9\%), followed by community-based program, 331 (34.7\%), then military-based program, 4 $(0.42 \%)$. The majority of residents trained in institutions that were located in an urban setting, 900 (94.3\%) versus nonurban, 54 (5.7\%). The Mid-West was the location for $288(30.2 \%)$ residents, followed by $248(26.0 \%)$ in the East, $236(24.7 \%)$ in the South, and $182(19.1 \%)$ in the West. There were 304 (31.9\%) PGY-1 residents, 286 (30.0\%) PGY-2 residents, and 364 (38.2\%) PGY-3 residents (Table 1). The difference in overall scores on the pretest questions based on PGY was not statistically significant (PGY-1: 56\% correct; PGY-2: 58\% correct; PGY-3: $55 \%$ correct; $p=0.08$ ), and also did not differ on grouped questions requiring comprehension and application of knowledge (PGY-1: 51\% correct; PGY-2: 55\% correct; PGY-3: 51\% correct; $p=0.06$ ).

\section{Correlation and reliability testing: attitude,}

preparedness, and behavior scales

The results from the PCA and Cronbach's alpha confirmed that the creation of the three scales (attitude, behavior, and preparedness) captured the intended information from the respective sets of questions. The 
Table 1. Characteristics of Resident Participants

\begin{tabular}{lr}
\hline Characteristic & $\boldsymbol{N}(\%)$ \\
\hline Residency program type & \\
$\quad$ Community & $331(34.7)$ \\
University & $619(64.9)$ \\
Military & $4(0.42)$ \\
Urban vs. nonurban location & \\
$\quad$ Nonurban & $54(5.7)$ \\
Urban & $900(94.3)$ \\
Region of United States & \\
East & $248(26.0)$ \\
Mid-West & $288(30.2)$ \\
South & $236(24.7)$ \\
West & $182(19.1)$ \\
Postgraduate level (PGY) & \\
PGY-1 & $304(31.9)$ \\
PGY-2 & $286(30.0)$ \\
PGY-3 & $364(38.2)$ \\
\hline
\end{tabular}

PCA yielded one factor with eigenvalues $>1.00$ and all respective items were found to load on one component, which confirmed the correlation of the respective set of questions. The Cronbach's alpha for items related to attitude, behavior, and preparedness was $0.80,0.86$, and 0.90 , respectively.

\section{Multiple linear regression outcomes}

In the regression model, a residents' attitude toward cross-cultural care was positively associated with pre- paredness (beta coefficient $[\beta]=0.57,95 \%$ confidence interval $[\mathrm{CI}]: 0.49-0.64, p<0.001)$. Both residents who reported serving a large number of racial/ethnic minorities and low-SES patients were positively associated with preparedness $(\beta=0.90,95 \%$ CI: $0.56-$ $1.24, p<0.001$ and $\beta=0.74,95 \%$ CI: $0.37-1.10$, $p<0.001$, respectively). The mean preparedness level was higher for residents who were at the PGY2 and PGY-3 levels than those at the PGY-1 level $(\beta=0.16,95 \%$ CI: $0.02-0.31, p=0.03$ and $\beta=0.18$, 95\% CI: $0.05-0.32, p=0.01$, respectively). Training at a university-based program was negatively associated with preparedness compared with training at a community-based program $(\beta=-0.15,95 \% \mathrm{CI}$ : -0.28 to $-0.02, p=0.03$; Table 2 ).

In the second linear model, behavior was negatively associated with knowledge ( $\beta=-0.40,95 \% \mathrm{CI}$ : -0.60 to $-0.20, p<0.001)$. Among residents with average preparedness, there was a significant positive relationship between attitude and knowledge $(\beta=0.51,95 \%$ CI: $0.32-0.70, p<0.001)$. A significant interaction between the residents' attitude level and preparedness was observed. As the level of preparedness increased, the positive association between attitude and knowledge decreased $(\beta=-0.15,95 \%$ CI: -0.22 to -0.08 , $p<0.001$; Table 3).

Table 2. Estimated Associations of Resident and Residency Program Characteristics with Self-Reported Preparedness $(N=954)$

\begin{tabular}{|c|c|c|c|}
\hline Characteristic & Beta-coefficient & $95 \% \mathrm{Cl}$ & $p$ \\
\hline Attitude scale $e^{a, b}$ & 0.57 & 0.49 to 0.64 & $<0.001$ \\
\hline Knowledge score (limited) ${ }^{b, c}$ & -0.03 & -0.11 to 0.06 & 0.57 \\
\hline Knowledge score (overall) ${ }^{\mathrm{b}, \mathrm{d}}$ & -0.01 & -0.08 to 0.05 & 0.73 \\
\hline Serves a large number of racial/ethnic minority patients ${ }^{b}$ & 0.90 & 0.56 to 1.24 & $<0.001$ \\
\hline Serves a large number of patients with a low-socioeconomic status ${ }^{b}$ & 0.74 & 0.37 to 1.10 & $<0.001$ \\
\hline \multicolumn{4}{|l|}{ Postgraduate level } \\
\hline PGY-1 & Reference & & \\
\hline PGY-2 & 0.16 & 0.02 to 0.31 & 0.03 \\
\hline PGY-3 & 0.18 & 0.05 to 0.32 & 0.01 \\
\hline \multicolumn{4}{|l|}{ Residency program type } \\
\hline Community & Reference & & \\
\hline University & -0.15 & -0.28 to -0.02 & 0.03 \\
\hline Military & 0.73 & -0.17 to 1.64 & 0.11 \\
\hline \multicolumn{4}{|l|}{ Region of United States } \\
\hline East & Reference & & \\
\hline Mid-West & -0.05 & -0.10 to 0.21 & 0.49 \\
\hline South & -0.06 & -0.23 to 0.10 & 0.46 \\
\hline West & -0.17 & -0.36 to 0.01 & 0.06 \\
\hline \multicolumn{4}{|l|}{ Urban vs. nonurban location } \\
\hline Nonurban & Reference & & \\
\hline Urban & 0.06 & -0.19 to 0.31 & 0.63 \\
\hline
\end{tabular}

Self-preparedness scale consisted of five validated questions that were grouped into a scale.

${ }^{a}$ The attitude scale consisted of five validated questions that were grouped into a scale.

${ }^{\mathrm{b}}$ All continuous variables were rescaled by dividing by 100 for ease of interpretation purposes.

'Knowledge score for the comprehension pretest questions only.

${ }^{d}$ Knowledge score for overall pretest questions. 
Table 3. Estimated Association of Behaviors, Attitudes, ${ }^{a}$ and Self-Reported Preparedness ${ }^{b}$ with Knowledge of Disparities ${ }^{c}$ Among Internal Medicine Residents $(N=954)$

\begin{tabular}{|c|c|c|c|}
\hline Characteristic & Beta-coefficient & $95 \% \mathrm{Cl}$ & $p$ \\
\hline Self-reported behavioral scale ${ }^{\mathrm{d}, e}$ & -0.40 & -0.60 to -0.20 & $<0.001$ \\
\hline Attitude scale ${ }^{\mathrm{a}, \mathrm{e}}$ & 0.51 & 0.32 to 0.70 & $<0.001$ \\
\hline Self-reported preparedness scale ${ }^{b, e}$ & 0.05 & -0.10 to 0.21 & 0.51 \\
\hline $\begin{array}{l}\text { Modification effect of self-reported } \\
\text { preparedness on attitude }\end{array}$ & -0.15 & -0.22 to -0.08 & $<0.001$ \\
\hline \multicolumn{4}{|l|}{ Postgraduate level } \\
\hline PGY-1 & Reference & & \\
\hline PGY-2 & 0.30 & -0.03 to 0.63 & 0.08 \\
\hline PGY-3 & 0.03 & -0.28 to 0.34 & 0.87 \\
\hline
\end{tabular}

aThe attitude scale consisted of five validated questions that were grouped into a scale.

${ }^{b}$ Self-preparedness scale consisted of six validated questions that were grouped into a scale.

${ }^{\mathrm{c}}$ Knowledge score for overall pretest questions.

${ }^{\mathrm{d}}$ The behavioral scale consisted of five validated questions that were grouped into a scale.

${ }^{\mathrm{e}}$ All of the above scales were rescaled by dividing by 100 for ease of interpretation purposes.

\section{Discussion}

Among residents studied, exposure to a greater volume of racial/ethnic minority and low-SES patients was associated with a more positive attitude toward cross-cultural care and greater perceived preparedness to deliver care to these populations. A positive attitude toward crosscultural care was also associated with better knowledge of related concepts. However, this knowledge did not improve during residency training. Also, preparedness to deliver cross-cultural care was found to be lower at university-based training programs than at community hospitals.

We also found an inverse relationship between clinical practice behaviors in cross-cultural care and knowledge. The relationship between attitude and knowledge was found to be negatively impacted by the effect of preparedness as well. The implications of these findings were unclear and could be better understood with further analysis on a more granular level.

Our findings that residents had poor knowledge of healthcare disparity-related concepts across PGY levels suggest that these concepts are not being taught during residency. Previous studies have shown that a number of providers continue to be unaware of the extent of healthcare disparities both nationally and in their own patient practices. ${ }^{10,27}$ Incorporating disparities education into cross-cultural curricula has the potential to better equip residents to deliver more equitable care to an increasingly racial and ethnic diverse patient population. $^{10,14}$

In the United States, residents care for a disproportionate share of racial and ethnic minority and low-SES patients, commonly at university-based programs. ${ }^{28}$ Yet, residents' self-reported preparedness to care for vulnerable patients was found to be lower for those training at university-based programs than for those training at community-based programs in this study. It was unclear whether these findings were more influenced by resident-, patient-, community-, or institutionallevel factors.

Residents who expressed less positive attitudes toward cross-cultural care and reported limited exposure to vulnerable patient populations felt less prepared to provide cross-cultural care. Residents are largely composed of young adults whose beliefs and moral values are well established. Cross-cultural care training may only have meaningful impact on a self-selected segment of residents who are open to developing their level of understanding and empathy for patients' social, economic, and cultural ecological context that influences their health. ${ }^{10}$ Curriculum need assessments may potentially aid in development of training that engages residents and shifts their attitudes toward a more patient-centered approach to delivering care. In addition, as healthcare moves toward value-based care, trainees may be more incentivize to engage in crosscultural care training. ${ }^{29}$

The findings in our secondary analysis were unclear. We found that residents who highly rated their clinical practice behaviors in caring for racial and ethnic minority and low-SES patients had lower scores on knowledge. Furthermore, we saw that residents' preparedness had a negative impact on the relationship between their attitude and knowledge. Taken together, these findings could suggest that residents who perceive themselves as prepared to deliver culturally sensitive care may lack awareness of their own knowledge gaps, because even in the setting of positive attitudes, 
they do not demonstrate increased knowledge. Objective assessments of cultural competence and better education during residency training can begin to address knowledge and potential performance gaps.

Our study had several limitations that could potentially affect its generalizability. First, the $52.8 \%$ response rate of participating programs represents residents from a selected group of training programs and may not fully represent residents at nonparticipating internal medicine residency programs. Also, program directors commonly select modules from the PEAC library for their residents to complete; some programs make the completion of modules compulsory, whereas others do not. However, our sample of programs was geographically diverse and included university-based and community-based hospitals, suggesting generalizability of results. We also relied on self-reported data regarding preparedness and clinical practice behavior, which may not be reflective of the residents' actual delivery or quality of care provided. ${ }^{30}$ Our study strengths included the use of validated questions, ${ }^{11,16}$ the creation of three unique scales related to attitude, preparedness (including care of lowliterate and low-SES patients), and behavior with domain content different from previously created cross-cultural scales, ${ }^{31}$ and the examination of the interaction of attitude and preparedness on knowledge.

\section{Conclusion}

Our study suggests that more training is needed to increase internal medicine residents' knowledge of cross-cultural care as it relates to health and healthcare disparities. This study speaks of the opportunity for programs to enhance how residents are trained to care for an ever increasing diverse patient population. Also, more research should be done to examine residents' perceived preparedness to deliver cross-cultural care at university-based programs, especially because these sites give service to large volumes of vulnerable populations. Our findings underline a need to better understand the role of residents' attitudes on preparedness to provide care for sociocultural diverse patients. Furthermore, it will be informative to explore how residents' actual provision of care for diverse patients relates to their attitude and knowledge of health and healthcare disparities.

\section{Acknowledgments}

Dr. Marshall had full access to all of the data in the study and takes responsibility for the integrity of the data and the accuracy of the data analysis. The study concept and design were by S.D.S., J.K.M., A.B., A.G., and L.A.C. Acquisition, analysis, or interpretation of data was by J.K.M., A.B., A.G., L.A.C., and S.D.S. Drafting of the article was by J.K.M. and S.D.S. Critical revision of the article for important intellectual content was done by all authors. Statistical analysis was by J.K.M. and A.B. Administrative, technical, or material support was by J.K.M., A.B., and N.M. Study supervision was by S.D.S. The authors would like to sincerely thank Dr. Brady West of the University of Michigan Center for Statistical Consultation and Research for his assistance with the statistical analysis. Dr. Sisson has received compensation from the Department of Medicine at the Johns Hopkins University for editing the article's featured curriculum. Dr. Cooper is supported by a grant from the National Heart, Lung, and Blood Institute (K24 HL083113).

\section{Author Disclosure Statement}

S.D.S has received compensation from the Department of Medicine at the Johns Hopkins University for editing the curriculum featured in this article. The other authors have no financial relationships to disclose and no conflicts of interest to report.

\section{References}

1. Institute of Medicine, Committee on Understanding and Eliminating Racial and Ethnic Disparities in Health Care; Smedley BD, Stith AY, Nelson AR, eds. Unequal Treatment: Confronting Racial and Ethnic Disparities in Health Care. Washington: National Academy Press, 2002.

2. Betancourt JR, Green AR, Carrillo JE, et al. Defining cultural competence: a practical framework for addressing racial/ethnic disparities in health and health care. Public Health Rep. 2003;118:293-302.

3. Carrillo JE, Green AR, Betancourt JR. Cross-cultural primary care: a patientbased approach. Ann Intern Med. 1999;130:829-834.

4. Betancourt JR, Cervantes MC. Cross-cultural medical education in the United States: key principles and experiences. Kaohsiung J Med Sci. 2009;25:471-478.

5. Betancourt JR, Green AR, Carrillo JE, et al. Cultural competence and health care disparities: key perspectives and trends. Health Aff (Millwood). 2005;24:499-505.

6. Institute of Medicine. Crossing the Quality Chasm: A New Health System for the 21st Century. Washington, DC: National Academy Press, 2001.

7. Betancourt JR. Cross-cultural medical education: conceptual approaches and frameworks for evaluation. Acad Med. 2003;78:560-569.

8. Champaneria MC, Axtell S. STUDENTJAMA. Cultural competence training in US medical schools. JAMA. 2004;291:2142.

9. Melamed E, Wyatt LE, Padilla T, et al. Patient-based cultural competency curriculum for pre-health professionals. Fam Med. 2008;40:726-733.

10. Smith WR, Betancourt JR, Wynia MK, et al. Recommendations for teaching about racial and ethnic disparities in health and health care. Ann Intern Med. 2007;147:654-665.

11. Weissman JS, Betancourt J, Campbell EG, et al. Resident physicians' preparedness to provide cross-cultural care. JAMA. 2005;294:1058-1067.

12. Greer JA, Park ER, Green AR, et al. Primary care resident perceived preparedness to deliver cross-cultural care: an examination of training and specialty differences. J Gen Intern Med. 2007;22:1107-1113.

13. Accreditation Council for Graduate Medical Education. ACGME program requirements for graduate medical education in internal medicine. ACGME approved categorization: 2012. Available at http:// 
www.acgme.org/portals/0/pfassets/programrequirements/140_internal_ medicine_2016.pdf Accessed June 1, 2016.

14. Brach C, Fraser I. Can cultural competency reduce racial and ethnic health disparities? A review and conceptual model. Med Care Res Rev. 2000;57 (Suppl 1):181-217.

15. Kern DE, Thomas PA, Hughes, MT. Curriculum Development for Medical Education: A Six-Step Approach. 2nd ed. Baltimore, MD: The Johns Hopkins University Press, 2009.

16. Braveman P, Egerter S, Williams DR. The social determinants of health: coming of age. Annu Rev Public Health. 2011;32:381-398.

17. Paez KA, Allen JK, Carson KA, et al. Provider and clinic cultural competence in a primary care setting. Soc Sci Med. 2008;66:1204-1216.

18. Sisson SD, Hughes MT, Levine D, et al. Effect of an internet-based curriculum on postgraduate education. A multicenter intervention. J Gen Intern Med. 2004;19(5 Pt 2):505-509.

19. Association of American Medical Colleges. Cultural competence education. 2005. Available at https://www.aamc.org/download/54338/data/ culturalcomped.pdf Accessed May 15, 2016.

20. World Health Organization. Social determinants of health. commission on social determinants of health-final report. Commission on social determinants of health, 2005-2008.

21. Centers for Disease Control and Prevention. The National Center for HIV/AIDS, Viral Hepatitis, STD, and TB Prevention. Social determinants of health. 2014. Available at https://www.cdc.gov/nchhstp/ socialdeterminants/definitions.html Accessed May 15, 2016.

22. Case SM, Swanson DB, ed. Constructing Written Test Questions for the Basic and Clinical Sciences. 3rd ed. Philadelphia, PA: National Board of Medical Examiners, 2002.

23. Paez KA, Allen JK, Beach MC, et al. Physician cultural competence and patient ratings of the patient-physician relationship. J Gen Intern Med. 2009;24:495-498.

24. The Society of General Internal Medicine. The society of general internal medicine: regional meetings. Available at http://www.sgim.org/ meetings/regional-meetings Accessed June 1, 2016.

25. U.S. Census Bureau. Geography. 2010 Census Urban and Rural Classification and Urban Area Criteria. Available at www.census.gov/geo/ reference/ua/urban-rural-2010.html Accessed April 6, 2016.
26. StataCorp. 2013. Stata Statistical Software: Release 13. College Station, TX: StataCorp LP.

27. Kaiser Family Foundation. Disparities Policy: Eliminating Racial/Ethnic Disparities in Health Care: What are the Options? 2008. Available at http:// kff.org/disparities-policy/issue-brief/eliminating-racialethnic-disparitiesin-health-care-what/\#back9 Accessed December 6, 2016.

28. American Medical Association. Why a healthy America needs resident physicians. 2013. Available at http://savegme.org/wp-content/uploads/ 2013/01/graduate-medical-education-action-kit.2 -3.pdf Accessed December 15, 2015.

29. Alberti PM, Bonham AC, Kirch DG. Making equity a value in value-based health care. Acad Med. 2013;88:1619-1623.

30. Johnson D, Cujec B. Comparison of self, nurse, and physician assessment of residents rotating through an intensive care unit. Crit Care Med. 1998;26:1811-1816.

31. Park ER, Chun MB, Betancourt JR, et al. Measuring residents' perceived preparedness and skillfulness to deliver cross-cultural care. J Gen Intern Med. 2009;24:1053-1056.

Cite this article as: Marshall JK, Cooper LA, Green AR, Bertram A Wright L, Matusko N, McCullough W, Sisson SD (2017) Residents' attitude, knowledge, and perceived preparedness toward caring for patients from diverse sociocultural backgrounds, Health Equity 1:1, 43-49, DOI: 10.1089/heq.2016.0010.
Abbreviations Used
PCA $=$ principal components analysis
PGY = postgraduate year
$\mathrm{SES}=$ socioeconomic status

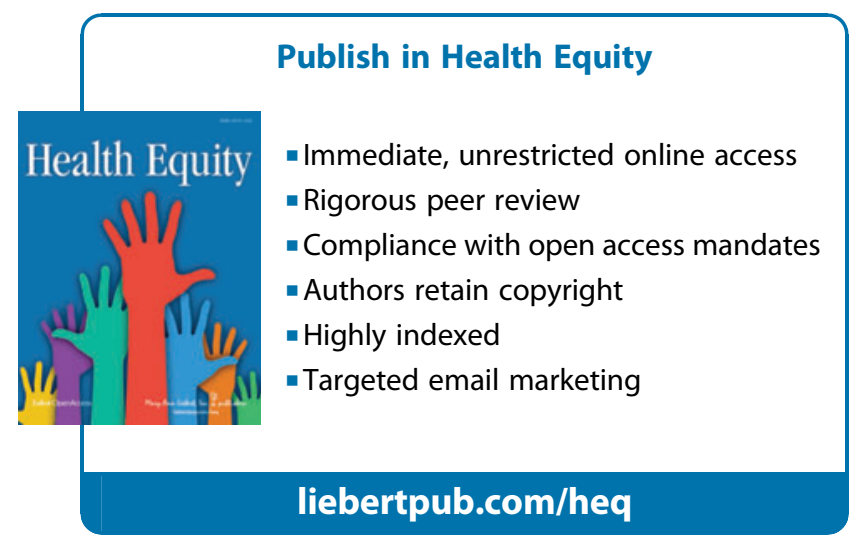

